This is an electronic reprint of the original article. This reprint may differ from the original in pagination and typographic detail.

Author(s): Luk, Hoi Ling; Feist, Johannes; Toppari, Jussi; Groenhof, Gerrit

Title: Multiscale Molecular Dynamics Simulations of Polaritonic Chemistry

Year: $\quad 2017$

Version:

Please cite the original version:

Luk, H. L., Feist, J., Toppari, J., \& Groenhof, G. (2017). Multiscale Molecular Dynamics Simulations of Polaritonic Chemistry. Journal of Chemical Theory and Computation, 13(9), 4324-4335. https://doi.org/10.1021/acs.jctc.7b00388

All material supplied via JYX is protected by copyright and other intellectual property rights, and duplication or sale of all or part of any of the repository collections is not permitted, except that material may be duplicated by you for your research use or educational purposes in electronic or print form. You must obtain permission for any other use. Electronic or print copies may not be offered, whether for sale or otherwise to anyone who is not an authorised user. 


\title{
Multi-scale Molecular Dynamics Simulations of Polaritonic Chemistry
}

\author{
Hoi Ling Luk, Johannes Feist, J. Jussi Toppari, and Gerrit Groenhof
}

J. Chem. Theory Comput., Just Accepted Manuscript • DOI: 10.1021/acs.jctc.7b00388 • Publication Date (Web): 27 Jul 2017

Downloaded from http://pubs.acs.org on July 31, 2017

\section{Just Accepted}

"Just Accepted" manuscripts have been peer-reviewed and accepted for publication. They are posted online prior to technical editing, formatting for publication and author proofing. The American Chemical Society provides "Just Accepted" as a free service to the research community to expedite the dissemination of scientific material as soon as possible after acceptance. "Just Accepted" manuscripts appear in full in PDF format accompanied by an HTML abstract. "Just Accepted" manuscripts have been fully peer reviewed, but should not be considered the official version of record. They are accessible to all readers and citable by the Digital Object Identifier (DOI@). "Just Accepted" is an optional service offered to authors. Therefore, the "Just Accepted" Web site may not include all articles that will be published in the journal. After a manuscript is technically edited and formatted, it will be removed from the "Just Accepted" Web site and published as an ASAP article. Note that technical editing may introduce minor changes to the manuscript text and/or graphics which could affect content, and all legal disclaimers and ethical guidelines that apply to the journal pertain. ACS cannot be held responsible for errors or consequences arising from the use of information contained in these "Just Accepted" manuscripts. 
Multi-Scale Molecular Dynamics Simulations of Polaritonic Chemistry

\author{
Hoi Ling Luk, ${ }^{\dagger}$ Johannes Feist, ${ }^{\ddagger}$ J. Jussi Toppari, ${ }^{\circledR}$ and Gerrit Groenhof ${ }^{*}, \dagger$ \\ $\dagger$ Nanoscience Center and Department of Chemistry, University of Jyväskylä, P.O. Box 35, 40014 \\ Jyväskylä, Finland. \\ $\ddagger$ Departamento de Física Teórica de la Materia Condensada and Condensed Matter Physics \\ Center (IFIMAC), Universidad Autónoma de Madrid Madrid, Spain. \\ ๆ Nanoscience Center and Department of Physics, University of Jyväskylä, P.O. Box 35, 40014 \\ Jyväskylä, Finland. \\ E-mail: gerrit.x.groenhof@jyu.fi
}

\begin{abstract}
When photoactive molecules interact strongly with confined light modes as found in plasmonic structures or optical cavities, new hybrid light-matter states can form, the so-called polaritons. These polaritons are coherent superpositions (in the quantum mechanical sense) of excitations of the molecules and of the cavity photon or surface plasmon. Recent experimental and theoretical works suggest that access to these polaritons in cavities could provide a totally new and attractive paradigm for controlling chemical reactions that falls in between traditional chemical catalysis and coherent laser control. However, designing cavity parameters to control chemistry requires a theoretical model with which the effect of the light-matter coupling on the molecular dynamics can be predicted accurately. Here we present a multi-scale quantum mechanics/molecular mechanics (QM/MM) molecular dynamics simulation model for photoactive molecules that are strongly coupled to confined light in optical cavities or surface plasmons. Using this model we have performed simulations with up to 1600 Rhodamine


molecules in a cavity. The results of these simulations reveal that the contributions of the molecules to the polariton are time-dependent due to thermal fluctuations that break symmetry. Furthermore, the simulations suggest that in addition to the cavity quality factor, also the Stokes shift and number of molecules control the lifetime of the polariton. Because large numbers of molecules interacting with confined light can now be simulated in atomic detail, we anticipate that our method will lead to a better understanding of the effects of strong coupling on chemical reactivity. Ultimately the method may even be used to systematically design cavities to control photochemistry.

\section{Keywords}

Strong light-matter coupling, polariton, cavity QED, excited states, quantum chemistry, molecular dynamics, QM/MM

\section{Introduction}

The goal of chemistry is to control reactions. To steer a reaction towards a desired product one needs to reshape the energy landscape of the molecules involved. Traditional chemical catalysts act on the transition states between ground-state free energy minima. Therefore, rational design of new catalysts requires detailed information of the ground-state potential energy surface. Quantum chemistry provides a convenient and cost-effective means to acquire such knowledge and has been used extensively to systematically develop and improve catalysts, ${ }^{1}$ including enzymes. ${ }^{2}$ For most, if not all, technologically relevant chemical processes catalysts are nowadays available.

In contrast, the possibilities to control the outcome of photochemical reactions with catalysts are still very limited. Yet, photochemistry is at the core of technologies for harvesting, converting and storing solar energy. Therefore, photonic catalysts that steer the excited-state dynamics towards the desired product state, while suppressing harmful side reactions, could be important to develop reliable low-cost photochemical solutions for a solar-fuelled economy.

Although photochemistry is sometimes used to induce reactions, for example in polymerization, ${ }^{3}$ catal- 
ysis of a photochemical process itself is notoriously difficult. Rather than acting on transitions between thermodynamic minima, such catalysts would need to act on the ultra-fast non-equilibrium molecular dynamics on electronic excited-state surfaces instead. So far only Nature has evolved efficient ways to control the outcome of a photochemical reaction. Prominent examples are the isomerization of the highly conjugated retinal co-factor in Rhodopsins, ${ }^{4}$ the harvesting of solar energy by light harvesting complexes ${ }^{5}$ and the highly directional charge separation in reaction centers. ${ }^{6}$

Photochemical reactions have been controlled with shaped laser pulses that drive the nuclear wave packet in a desired direction through the formation of a superposition of vibrational excitations. ${ }^{7}$ However, despite advances in quantum chemistry software and computer hardware, the object functions for optimal control with weak-field laser pulses are too complicated to be solved for anything but the simplest chemical systems. Instead, successful applications of coherent control on complex systems therefore rely on genetic algorithms to search for the optimal pulse shape,${ }^{8}$ a process that does not provide insight into the process that is the target of the optimization. These limitations prevent scaling up of coherent laser control techniques for technological applications.

Strong laser fields can also be used to reshape the excited state potential energy landscape and steer a reaction. ${ }^{9}$ However, the high intensities required to generate sufficiently strong fields also induces radiation damage due to the absorption of multiple photons. Because confining light to smaller volumes increases the field strength per photon, structures that confine light, such as optical cavities, plasmonic particles or surface plasmons, could in principle be used to shape the energy landscape with low intensities. Indeed, in a series of experiments on photo-reactive molecules in optical cavities Ebbesen and others have shown that the interaction with confined light changes their photochemistry in case of photo-isomerization ${ }^{10}$ and energy transport. ${ }^{11,12}$ Although it is not understood how the interaction with the confined light affects the reaction mechanisms, these observations suggest that catalysis with cavities could provide a totally new and attractive paradigm for controlling photochemical reactions that falls somewhere in between traditional chemical catalysis and coherent laser control. ${ }^{13}$

Controlling photochemistry with confined light requires a much better understanding of the effects of strong coupling on the molecular dynamics than presently exists. Because the relevant time and spatial resolutions are notoriously difficult to access in experiments, theoretical models with which these effects can be investigated in atomistic detail will be essential to unlock the potential of photochemistry with confined 
light.

While initial theoretical studies of strong coupling treated molecules as two-level systems, several theoretical models that include also nuclear degrees of freedom, have been developed in the last years. These were limited, however, to treating vibrational modes within the harmonic oscillator approximation, ${ }^{14-16}$ which can not describe most chemical reactivity. We recently showed how to apply the well-known BornOppenheimer approximation in the case of strong coupling, leading to a description of nuclear motion on polaritonic (hybrid light-matter) potential energy surfaces. ${ }^{17,18}$ Up to now, this approach was only applied to simplified one-dimensional model molecules, but here we extend it to allow a full atomistic treatment of realistic molecules based on hybrid Quantum Mechanics/Molecular Mechanics (QM/MM) molecular dynamics simulations. ${ }^{19,20}$

\section{Theoretical Background}

The conceptually simplest structure to confine light in is the Fabry-Pérot optical micro-cavity with two mirrors (Figure 1). These mirrors can consist of a thin metal film or of alternating layers of high and low dielectric materials (i.e. dielectric or Bragg mirror). Only photons with a wavelength $\left(\lambda_{\text {cav }}\right)$ that matches the condition for constructive interference between the mirrors (i.e. $2 d \approx n \lambda_{\text {cav }}$ with $n \in \mathbb{N}$ ) can become trapped inside the cavity and 'bounce' back and forth. Eventually, this photon is lost due to absorption in or tunneling through the mirrors or to other decay processes. The total lifetime of a photon in a cavity $\left(\tau_{\text {cav }}\right)$ determines the quality, or Q-factor, of that cavity:

$$
\mathrm{Q}=\frac{\omega_{\mathrm{cav}}}{\gamma_{\mathrm{cav}}}
$$

Here $\gamma_{\text {cav }}$ is the decay rate $\left(1 / \tau_{\text {cav }}\right)$ of the cavity photon with energy $\hbar \omega_{\text {cav }}$. The cavity Q-factor depends on the accuracy of the nanofabrication process, the thickness of the mirrors and the cavity materials.

To present a self-contained description of our multi-scale approach, we start from the established model for two-level emitters inside optical cavities ${ }^{21}$ that provides a good approximation for atoms. For a single emitter (e.g. an atom) with an electronic ground $|g\rangle$ and excited state $|e\rangle$ in a cavity that can confine a photon of energy $\hbar \omega_{\text {cav }}$, the total Hamiltonian consists of the atomic excitation $\left(\hat{H}_{\mathrm{a}}\right)$, cavity excitation $\left(\hat{H}_{\text {cav }}\right)$ and 


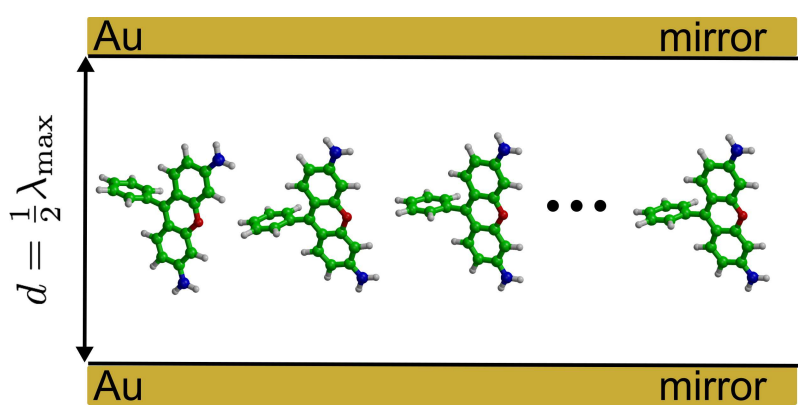

Figure 1: Schematic illustration of a Fabry-Pérot cavity with Rhodamine molecules inside (not to scale!).

the light-matter interaction $\left(\hat{H}_{\text {int }}\right)$ :

$$
\hat{H}_{\text {tot }}=\hat{H}_{\mathrm{a}}+\hat{H}_{\mathrm{cav}}+\hat{H}_{\text {int }}
$$

Using the Rabi quantum model for an atom interacting with a quantized electromagnetic field, this Hamiltonian can be written as:

$$
\hat{H}_{\text {tot }}=\hbar \omega_{\mathrm{a}} \hat{\sigma}^{\dagger} \hat{\sigma}+\hbar \omega_{\mathrm{cav}} \hat{a}^{\dagger} \hat{a}+\hbar g\left(\hat{a}^{\dagger}+\hat{a}\right)\left(\hat{\sigma}^{\dagger}+\hat{\sigma}\right)
$$

Here $\hbar \omega_{\mathrm{a}}$ is the excitation energy of the atom (i.e., energy difference between $|e\rangle$ and $|g\rangle$ ) and $\hbar \omega_{\text {cav }}$ the energy of the cavity photon. The operator $\hat{\sigma}^{\dagger}(\hat{\sigma})$ creates (annihilates) the excitation of the atom, while $\hat{a}^{\dagger}$ $(\hat{a})$ creates (annihilates) a photon in the cavity. The strength of the light-matter interaction, $\hbar g$, is calculated within the dipole approximation as the overlap between the transition dipole $\left(\boldsymbol{\mu}^{e \rightarrow g}\right)$ for the excitation of the atom and the electric component of the cavity photon:

$$
\hbar g=\boldsymbol{\mu}^{e \rightarrow g} \cdot \mathbf{u}_{\mathrm{cav}} \sqrt{\hbar \omega_{\mathrm{cav}} / \epsilon_{0} V_{\mathrm{cav}}}
$$

Here $V_{\text {cav }}$ is the effective mode volume of the cavity, in which the photon with frequency $\omega_{\text {cav }}$ (the energy $\left.\hbar \omega_{\text {cav }}\right)$ is confined, $\epsilon_{0}$ the vacuum permittivity and $\mathbf{u}_{\mathrm{cav}}$ the unit vector indicating the direction of the electric component of the confined mode.

If the interaction is weak, only the radiative properties of the atom are affected. This is known as the Purcell effect ${ }^{22}$ and can be described with first order perturbation theory. If, however, the interaction is so strong that coherent exchange of energy between the atom and electro-magnetic field in the cavity becomes 


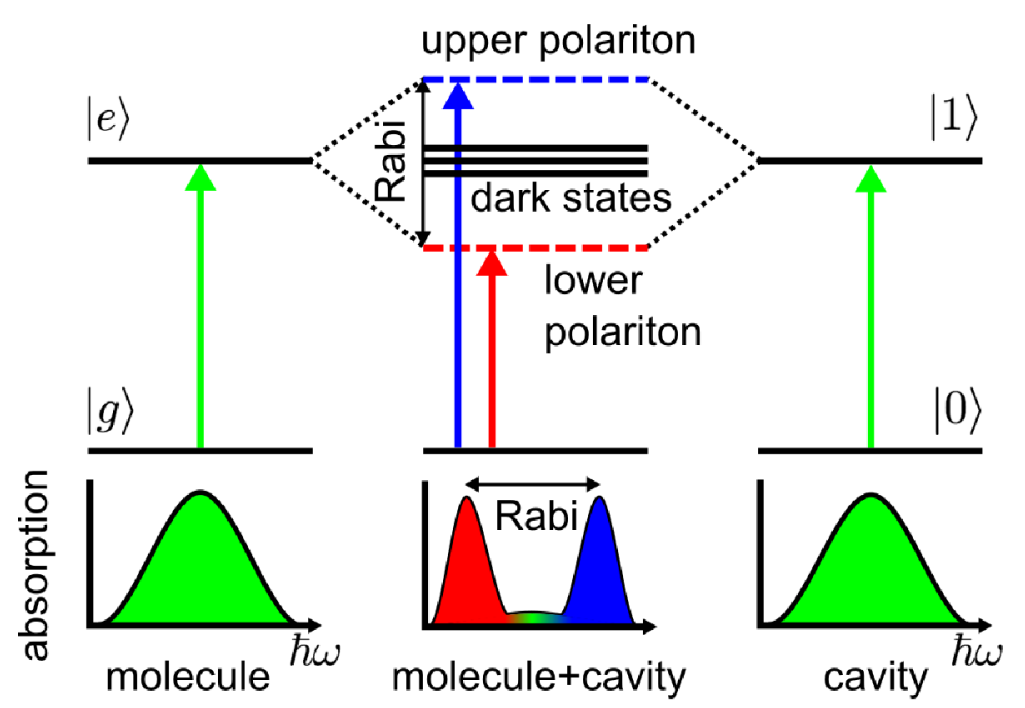

Figure 2: Schematic illustration of strong coupling between a confined photon and molecules (atoms) in an optical cavity. If tuned, that is the absorption maxima of the molecules and cavity coincide, the absorption splits into two peaks separated by the Rabi splitting. The new states associated with these peaks are the upper (blue) and lower (red) polaritons that are superpositions of the molecular and cavity excitation. If there is more than one molecule (atom) in the cavity, there are also dark states that have little or no contribution from the cavity photon and therefore are at the same energy level as the excited state of the molecule but with very low oscillator strength.

faster than the decay processes, new hybrid light-matter states form. ${ }^{23}$ This is the strong-coupling limit and will be the focus of our work. We note that in the regimes of ultrastrong and deep strong coupling where the coupling strengths become comparable to the transition frequencies, additional self-interaction terms should be included in Equation 3. ${ }^{24-26}$

In the strong coupling regime, the solution to the light-matter Hamiltonian is difficult to obtain without approximations. Neglecting the fast oscillating terms $\hat{a}^{\dagger} \hat{\sigma}^{\dagger}$ and $\hat{a} \hat{\sigma}$ is valid if the optical transition in the atom and cavity photon are near resonance (i.e. similar) and the coupling strength $(\hbar g)$ is significantly smaller than the excitation energy. This is the rotating wave approximation (RWA). With these approximations, the expression that remains is known as the Jaynes-Cummings model: ${ }^{27}$

$$
\hat{H}_{\mathrm{JC}}=\hbar \omega_{\mathrm{a}} \hat{\sigma}^{\dagger} \hat{\sigma}+\hbar \omega_{\mathrm{cav}} \hat{a}^{\dagger} \hat{a}+\hbar g\left(\hat{a}^{\dagger} \hat{\sigma}+\hat{a} \hat{\sigma}^{\dagger}\right)
$$


Within the single-excitation subspace (i.e. a single photon in the cavity or atom), the solutions to the JaynesCummings Hamiltonian are coherent superpositions of the electronic excitation in the atom and of the excitation in the cavity (i.e. presence of a photon):

$$
\begin{aligned}
& \Psi_{\mathrm{LP}}=\alpha_{\mathrm{LP}}|g\rangle|1\rangle+\beta_{\mathrm{LP}}|e\rangle|0\rangle \\
& \Psi_{\mathrm{UP}}=\alpha_{\mathrm{UP}}|g\rangle|1\rangle+\beta_{\mathrm{UP}}|e\rangle|0\rangle
\end{aligned}
$$

These new hybrid light-atom states are called the lower $\left(\Psi_{\mathrm{LP}}\right)$ and upper polariton $\left(\Psi_{\mathrm{UP}}\right)$ and are linear combinations of the uncoupled wave-function product of the atom in the ground state $(|g\rangle)$ with one photon in the cavity $(|1\rangle)$, and of the uncoupled wave function product of the atom in the excited state $(|e\rangle)$ without photon $(|0\rangle)$, with coefficients $\alpha$ and $\beta\left(\alpha^{2}+\beta^{2}=1\right)$. The energies of these polaritons are:

$$
E_{\mathrm{UP} / \mathrm{LP}}=\frac{1}{2} \hbar\left(\omega_{\mathrm{a}}+\omega_{\mathrm{cav}}\right) \pm \hbar \sqrt{\frac{1}{4}\left(\omega_{\mathrm{a}}-\omega_{\mathrm{cav}}\right)^{2}+g^{2}}
$$

The polaritons deviate from the uncoupled states when the energies of the cavity photon and atomic excitation are similar. The maximal deviation occurs at resonance, when the energies of the uncoupled states are the same ('tuned', as in Figure 2). In this situation the energy gap between the polaritonic states is minimal and called the Rabi splitting: $\hbar \Omega_{\text {Rabi }}=E_{\mathrm{UP}}-E_{\mathrm{LP}}$. Because of Rabi splitting, the absorption spectrum of the atom plus cavity has two peaks instead of one, located below and above the absorption maxima of the 'bare' cavity and atom (Figure 2). The Rabi splitting is sometimes referred to as the vacuum Rabi splitting to indicate that the polariton states exist even without a photon, but obviously are not populated then.

Tavis and Cummings extended this model to many $(N)$ two-level atoms. ${ }^{28}$ The eigenstates in the singleexcitation subspace are now superpositions of all uncoupled states in which either a single emitter is excited (with all other emitters in the ground state, and no photon in the cavity), or a single photon is in the cavity (and all emitters in their ground state):

$$
\Psi_{K}=\sum_{i=1}^{N} \beta_{i}^{K}\left|g_{1} g_{2} . . e_{i} . . g_{N-1} g_{N}\right\rangle|0\rangle+\alpha^{K}\left|g_{1} g_{2} . . g_{i} . . g_{N-1} g_{N}\right\rangle|1\rangle
$$

The index $K$ labels the $N+1$ eigenstates of the system. If all atoms are identical $\left(\hbar \omega_{a, i}=\hbar \omega_{a}\right.$ and $\left.g_{i}=g\right)$, 
these eigenstates again contain an upper and a lower polariton, formed by a superposition of the molecular 'bright' state $|B\rangle=1 / \sqrt{N} \sum_{i}\left|g_{1} g_{2} . . e_{i} . . g_{N-1} g_{N}\right\rangle|0\rangle$ with the single-photon state $\left|g_{1} g_{2} . . g_{N}\right\rangle|1\rangle$, while all $N-1$ other superpositions of molecular excitations are 'dark' states that do not couple to the photon mode $\left(\alpha^{K}=0\right)$, and are not visible in the spectrum (Figure 2). The energy gap between the upper and lower polaritonic states (Rabi splitting) increases with a factor $\sqrt{N}$ :

$$
\hbar \Omega_{\mathrm{Rabi}}^{\max }=2 \sqrt{N} \hbar g
$$

In contrast, if the atoms are not identical and their excitations are inhomogeneously broadened, the 'dark' states also contain a photon component, albeit smaller than in the two 'bright' states. Therefore, in reality these 'dark' states absorb weakly as shown for example in Figure 2 of Hourdré et al. ${ }^{29}$

Although the Jaynes-Cummings and Tavis-Cummings models are only valid for two-level emitters in cavities, these models are used frequently to rationalize the effect of strong coupling between molecules and confined light. ${ }^{23}$ Whereas these models can capture the effect of the coupling on the spectrum or on the delocalization of the excitation energy, in particular for quantum dots or molecules at cryogenic temperatures, they cannot predict nor rationalize the effect on chemical reactivity due to the neglect of the molecular degrees of freedom.

Both Jaynes-Cummings and Tavis-Cummings models have therefore been extended to di- and triatomic molecules and used to investigate the effect of one or two internal degrees of freedoms. ${ }^{16-18,25,26,30-34}$ These low dimensional molecular models have provided important insights into the dynamics under strong coupling, such as correlations between molecular motions in distant molecules, ${ }^{17}$ suppression of vibronic progression ${ }^{16}$ and effects on population transfer at avoided crossings, ${ }^{31,34}$ but have limited predictive power for condensed-phase dynamics of molecules with many degrees of freedom. In order to deepen our understanding of the effects of strong coupling on molecular dynamics and chemical reactivity, we therefore develop a fully atomistic model that describes the high-dimensional molecular energy landscape and can represent the effects of environment, temperature and pressure.

A popular technique for simulating molecules in atomic detail is molecular dynamics (MD). Since its introduction more than forty years ago, ${ }^{35,36}$ advances in computer hardware, algorithmic developments and improvement of interaction functions, have made MD into a powerful predictive technique. ${ }^{37}$ In previous 
work we have developed a multi-scale MD model for photo-reactivity in complex systems. ${ }^{38}$ A quantum mechanical description is used to model the electronic rearrangement for those parts of the system that are involved in the absorption of the photon, usually the chromophore. For the remainder a simple force field model suffices. The interactions in the system are thus computed within the multi-scale quantum/classical framework (QM/MM) introduced by Warshel and Levitt over four decades ago. ${ }^{19}$ Forces are calculated onthe-fly, and a surface hopping algorithm is used to model radiationless transitions between electronic states during the dynamics. ${ }^{39}$ Using femtosecond time-resolved x-ray crystallography at a free electron laser, we have confirmed that this multi-scale model correctly captures the dynamics and reactivity in the optically excited state of the photoactive yellow protein (PYP), ${ }^{40,41}$ a bacterial photoreceptor. Here, we adapt this approach to model molecular dynamics on hybrid light-matter (i.e. polaritonic) potential energy surfaces ${ }^{17}$ to perform atomistic simulations of photoactive molecules strongly coupled to confined light modes inside optical cavities. We exploit that polaritons are superpositions of molecular ground and excited states, both of which are described accurately in this multi-scale MD approach, ${ }^{38}$ and use the existing strategies to deal with the new non-adiabatic hopping terms between these hybrid surfaces. ${ }^{17}$

\section{Methodology}

Under the assumption that the electrons and cavity photon can adapt instantaneously to displacements of the nuclei, we separate the nuclear degrees of freedom from the much faster electronic plus photonic degrees of freedom. ${ }^{17}$ As a consequence, the energy levels of the Tavis-Cummings Hamiltonian ${ }^{28}$ are now functions of the nuclear coordinates $(\mathbf{R}(t))$ and form the hybrid light-matter potential energy surfaces on which the nuclei move. Because of the nuclear dynamics, the eigenstates of Tavis-Cummings Hamiltonian not only depend on the nuclear coordinates, but also on time, i.e., $\alpha^{K}[\mathbf{R}(t)]$ and $\beta_{i}^{K}[\mathbf{R}(t)]$.

The multi-dimensional light-matter Hamiltonian for $N$ molecules, including environment (a total of $n$ 
atoms per molecule) is:

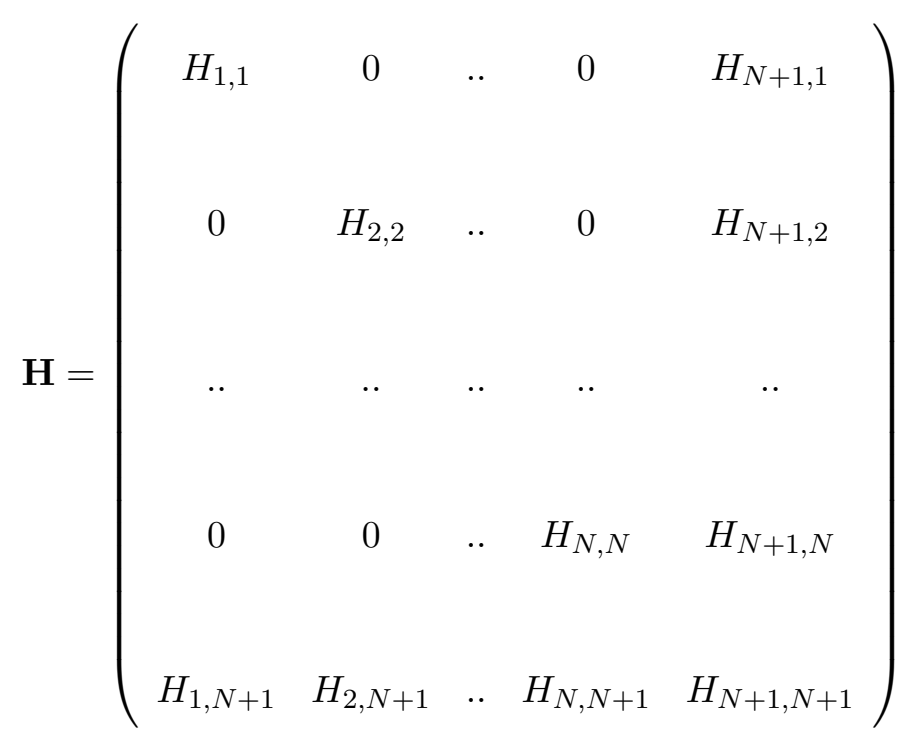

where the elements are functions of the $3 n$ atomic coordinates of all $(N)$ molecules with their environments and can be computed at the QM/MM level. The diagonal elements are the usual 'bare' electronic QM/MM energies of the ground $\left(\mathrm{S}_{0}\right)$ or excited states $\left(\mathrm{S}_{1}\right)$. The first $N$ diagonal matrix elements represent the cases in which one $(i)$ of the $N$ molecules is excited, while the other molecules $(j \neq i$ with $1 \leq j \leq N)$ are in the ground state:

$$
\begin{aligned}
& H_{i, i}= \\
& \left\langle 0\left|\left\langle g_{1}\left(\mathbf{R}_{1}\right) g_{2}\left(\mathbf{R}_{2}\right) . . e_{i}\left(\mathbf{R}_{i}\right) . . g_{N-1}\left(\mathbf{R}_{N-1}\right) g_{N}\left(\mathbf{R}_{N}\right)|\hat{H}| g_{1}\left(\mathbf{R}_{1}\right) g_{2}\left(\mathbf{R}_{2}\right) . . e_{i}\left(\mathbf{R}_{i}\right) . . g_{N-1}\left(\mathbf{R}_{N-1}\right) g_{N}\left(\mathbf{R}_{N}\right)\right\rangle\right| 0\right\rangle
\end{aligned}
$$

with $1 \leq i \leq N$. $\mathbf{R}_{i}$ is the $3 n$ dimensional vector of all atomic positions in molecule $i$, including its (solvent) environment. Under the assumption that direct interactions between the photoactive molecules can be neglected, $\hat{H}$ is a sum of $N$ independent molecular QM/MM Hamiltonians:

$$
\hat{H}=\sum_{k}^{N} \hat{h}^{\mathrm{QM} / \mathrm{MM}}\left(\mathbf{R}_{k}\right)
$$

The eigenstates of $\hat{h}^{\mathrm{QM} / \mathrm{MM}}\left(\mathbf{R}_{k}\right)$ are the electronic ground states $\left|g_{j}\left(\mathbf{R}_{j}\right)\right\rangle$ of molecules $k=j \neq i$ and 
electronic excited state $\left|e_{i}\left(\mathbf{R}_{i}\right)\right\rangle$ of molecule $k=i$ and are added together to give the total energy:

$$
H_{i, i}=V_{\mathrm{S}_{1}}^{\mathrm{QM} / \mathrm{MM}}\left(\mathbf{R}_{i}\right)+\sum_{j \neq i}^{N} V_{\mathrm{S}_{0}}^{\mathrm{QM} / \mathrm{MM}}\left(\mathbf{R}_{j}\right)
$$

These energies (and gradients) are readily calculated at QM/MM level, using for example the complete active space self-consistent field (CASSCF), ${ }^{42}$ Configuration Interaction with Single (CIS) or time-dependent Density functional theory (TDDFT) ${ }^{43}$ approaches for the QM subsystem. Direct interactions between the molecules are ignored here, but can be included at additional computational costs if the QM/MM energy of molecule $i\left(V^{\mathrm{QMMM}}\left(\mathbf{R}_{i}\right)\right)$ is evaluated in the field of point charges fitted to electron density of the other molecules, as in divide-and-conquer QM/MM approaches. ${ }^{44}$

The element $H_{N+1, N+1}$ models the situation in which all molecules are in the electronic ground state, while there is one photon in the optical cavity with energy $\hbar \omega_{\text {cav }}$ :

$$
\begin{aligned}
H_{N+1, N+1} & =\left\langle 1\left|\left\langle g_{1}\left(\mathbf{R}_{1}\right) g_{2}\left(\mathbf{R}_{2}\right) . . g_{i}\left(\mathbf{R}_{i}\right) . . g_{N}\left(\mathbf{R}_{N}\right)|\hat{H}| g_{1}\left(\mathbf{R}_{1}\right) g_{2}\left(\mathbf{R}_{2}\right) . . g_{i}\left(\mathbf{R}_{i}\right) . . g_{N}\left(\mathbf{R}_{N}\right)\right\rangle\right| 1\right\rangle \\
& =\sum_{i}^{N}\left\langle g_{i}\left(\mathbf{R}_{i}\right)\left|\hat{h}^{\mathrm{QM} / \mathrm{MM}}\left(\mathbf{R}_{i}\right)\right| g_{i}\left(\mathbf{R}_{i}\right)\right\rangle+\hbar \omega_{\text {cav }} \\
& =\sum_{i}^{N} V_{\mathrm{S}_{0}}^{\mathrm{QM} / \mathrm{MM}}\left(\mathbf{R}_{i}\right)+\hbar \omega_{\text {cav }}
\end{aligned}
$$

The off-diagonal terms describe the interactions with the cavity photon and are approximated as the overlap between the transition dipole moment of the excited molecule (computed at the QM/MM level) and the electric field of the confined photon, as in the Jaynes-Cummings model: ${ }^{27}$

$$
H_{N+1, i}=H_{i, N+1}=\boldsymbol{\mu}_{i}^{g \rightarrow e}\left(\mathbf{R}_{i}\right) \cdot \mathbf{u}_{\mathrm{cav}} \sqrt{\hbar \omega_{\mathrm{cav}} / \epsilon_{0} V_{\mathrm{cav}}}
$$

with $\mathbf{u}_{\mathrm{cav}}$ the unit vector pointing in the direction of the electric field of the confined photon at the position of the molecule. For optical mirco-cavities, variations in the field strength over the dimension of the molecule can be safely neglected, because the molecules are much smaller than the cavity. Excitonic interactions between the photoactive molecules are neglected here, which is valid for low concentrations of photoactive molecules. However, for simulations at higher concentrations, these excitonic interactions can be included 
at additional computational cost through a multi-pole expansion of the molecular transition densities, as is commonly done in exciton models. ${ }^{45}$

The matrix is diagonalized at every MD step to yield the $N+1$ (adiabatic) hybrid states (Equation 8 , Figure 2). Trajectories of all atoms in the system are computed by numerically integrating Newton's equations of motion associated with the potential energy surface of one of these states. The forces acting on the atoms of all molecules in the system are computed by invoking the Hellmann-Feynman theorem. For atom $a$ of molecule $i$ in state $K$, the Hellmann-Feynman force is (assuming the coefficients are real):

$$
\begin{aligned}
\mathbf{F}_{a \in i}^{K}= & -\left\langle\Psi^{K}\left|\nabla_{a \in i} \mathbf{H}\right| \Psi^{K}\right\rangle \\
= & -\left(\beta_{i}^{K}\right)^{2} \nabla_{a \in i}\left\langle e_{i}\left(\mathbf{R}_{i}\right)\left|\hat{h}^{\mathrm{QM} / \mathrm{MM}}\left(\mathbf{R}_{i}\right)\right| e_{i}\left(\mathbf{R}_{i}\right)\right\rangle \\
& -\nabla_{a \in i}\left\langle g_{i}\left(\mathbf{R}_{i}\right)\left|\hat{h}^{\mathrm{QM} / \mathrm{MM}}\left(\mathbf{R}_{i}\right)\right| g_{i}\left(\mathbf{R}_{i}\right)\right\rangle\left[\left(\alpha^{K}\right)^{2}+\sum_{j \neq i}^{N}\left(\beta_{j}^{K}\right)^{2}\right] \\
& -\alpha^{K} \beta_{i}^{K} \nabla_{a \in i}\left\langle g_{i}\left(\mathbf{R}_{i}\right)\left|\boldsymbol{\mu}_{i}^{g \rightarrow e}\left(\mathbf{R}_{i}\right)\right| e_{i}\left(\mathbf{R}_{i}\right)\right\rangle \cdot \mathbf{u}_{\mathrm{cav}} \sqrt{\hbar \omega_{\mathrm{cav}} / \epsilon_{0} V_{\mathrm{cav}}} \\
& -\beta_{i}^{K} \alpha^{K} \nabla_{a \in i}\left\langle e_{i}\left(\mathbf{R}_{i}\right)\left|\boldsymbol{\mu}_{i}^{g \rightarrow e}\left(\mathbf{R}_{i}\right)\right| g_{i}\left(\mathbf{R}_{i}\right)\right\rangle \cdot \mathbf{u}_{\mathrm{cav}} \sqrt{\hbar \omega_{\mathrm{cav}} / \epsilon_{0} V_{\mathrm{cav}}}
\end{aligned}
$$

There are several options to correct the breakdown of this 'light-matter Born-Oppenheimer approximation' when the energy gaps between the hybrid states are small. ${ }^{17}$ Here, we use diabatic surface hopping, based on the Landau-Zener model, ${ }^{46,47}$ which relates the probability of a transition between two states $\Psi^{K}$ and $\Psi^{L}$ to the non-adiabatic coupling, via

$$
P_{K \rightarrow L}=\exp \left(-\frac{1}{4} \pi \xi\right)
$$

with $\xi$ the Massey parameter, defined as: ${ }^{48}$

$$
\xi=\frac{\Delta E^{K L}}{\hbar\left\langle\Psi^{K} \mid \frac{\partial}{\partial t} \Psi^{L}\right\rangle} .
$$

Following Hammes-Schiffer and Tully, ${ }^{49}$ we approximate the non-adiabatic coupling $\left\langle\Psi^{K} \mid \frac{\partial}{\partial t} \Psi^{L}\right\rangle$ as $\left\langle\Psi^{K}(t)\right| \Psi^{L}(t+$ 
Calculating this overlap and the energy gap $\Delta E^{K L}$ at every time step is straightforward, and we can use the Landau-Zener formula to calculate the probability of a transition to the other surface (Equation 17). In principle, this transition probability could be used to spawn a new trajectory on the other surface, but since this procedure would lead to multiple trajectories that have to be computed simultaneously, spawning will be too demanding in practice. Instead, we therefore restrict hopping to situations where the transition probability approaches unity. This happens when the states $K$ and $L$ are degenerate: $\Delta E^{K L} \approx 0$.

By restricting the hopping to these degeneracies, the classical trajectories never leave the diabatic surface. Therefore, energy and momentum are conserved. Furthermore, we avoid the computationally expensive calculation of the $\frac{1}{2} N(N+1)$ non-adiabatic coupling vectors between all pairs of states. However, because this strict diabatic hopping criterion could lead to an underestimation of the population transfer probability, ${ }^{39}$ our model is clearly an approximation, but can provide photochemical mechanisms in agreement with experiment. ${ }^{40,41}$

Spontaneous decay of the cavity photon $\left(\gamma_{\text {cav }}\right)$ or radiationless deactivation of one of the molecules at a conical intersection are also included in the model. The final state of these processes has all molecules in their electronic ground $\left(\mathrm{S}_{0}\right)$ states and no photon in the cavity: $\left|g_{1} g_{2}, . . g_{N}\right\rangle|0\rangle$. This term was intentionally omitted from the Hamiltonian above (Equation 10), because the coupling to this final state is described differently from the light-matter couplings. Decay of the cavity photon can be included by stochastic jumps to the ground state. The probability for such jumps are calculated with the cavity Q-factor, which is a parameter in the simulation, and the weight of the cavity photon in the hybrid state: $P^{K \rightarrow 0}(t)=\left|\alpha^{K}(t)\right|^{2} \gamma_{\text {cav }} \Delta t$. Because for photoreactive molecules the non-adiabatic coupling between the excited $\left(S_{1}\right)$ and ground $\left(S_{0}\right)$ electronic states of the molecule is only strong enough near surface crossings to induce radiationless transitions, these coupings are very localized in phase space. Therefore, also these couplings are not included 

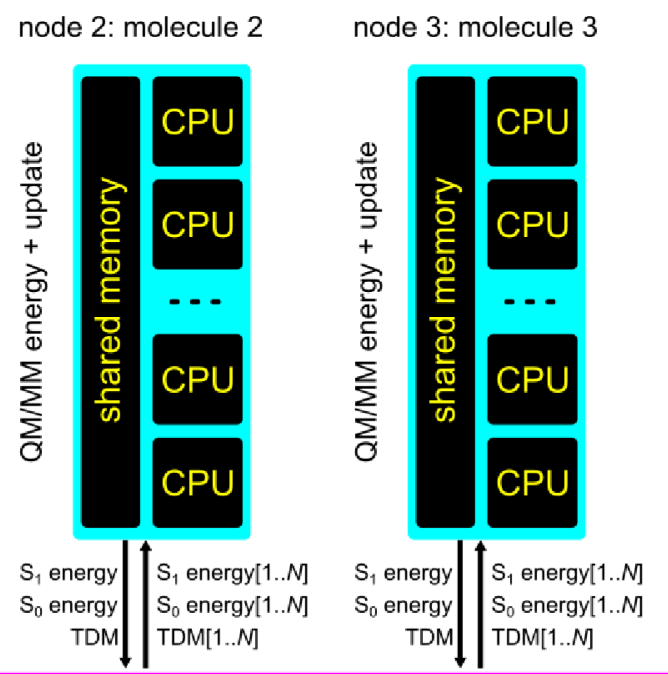

node $N$ : molecule $N$

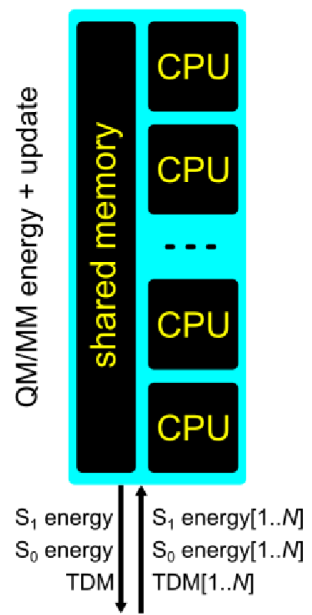
interconnect: MPI

Figure 3: Schematic illustration of the parallel cavity molecular dynamics implementation in Gromacs. Each molecule is assigned to a compute node that uses all available CPUs (or GPUs) to compute the QM/MM energies and gradients of the electronic ground and excited states, as well as the transition dipole moment. The energies and dipole moments are sent to all other nodes. After these double-precision numbers are received, the nodes diagonalize the light-matter Hamilton matrix (Equation 10), calculate the forces (Equation 16) and update the coordinates.

explicity in the Hamiltonian (Equation10) but are treated in an ad hoc manner instead by means of surface hopping, as in our previous work. ${ }^{38,50}$

We have implemented the multi-scale model for polariton chemistry in the open source MD program Gromacs, version 4.5. ${ }^{51}$ Because the excited and ground state energies and the transition dipole moments can be computed for each molecule on a separate computer node, while communication and storage are limited to only $3 N$ double precision numbers per MD step, the code scales optimally on parallel computers (Figure 3). Therefore, on modern supercomputers, very large system sizes can be simulated. Indeed, we have tested the code with up to 1,600 molecules on a Cray XC40 and ran simulations with 43,200 QM and $17,700,800 \mathrm{MM}$ atoms, using $38,400 \mathrm{CPU}$ cores in parallel. 


\section{Simulation details}

In this work, we use the 'bare' Rhodamine model shown in Figure 4. A force field, based on amber03 was constructed. ${ }^{52}$ Charges were derived with a two-stage RESP fit ${ }^{53}$ to the electron density calculated at the B3LYP/cc-pVTZ level of theory ${ }^{54,55}$ with the Gaussian09 program. ${ }^{56}$ Charges and atomtypes are included as Supporting Information. After a 200 steps steepest descent energy minimization, the Rhodamine was placed at the center of a rectangular box and filled with 3684 TIP3P water molecules. ${ }^{57}$ After 2 ns of equilibration with harmonic position restraints on the heavy atoms of the Rhodamine molecule (force constant $1000 \mathrm{kJmol}^{-1} \mathrm{~nm}^{-2}$ ), a $200 \mathrm{~ns}$ classical Molecular Dynamics trajectory was computed at constant temperature $(300 \mathrm{~K})$ using a stochastic dynamics integrator with a friction coefficient of $0.1 \mathrm{ps}^{-1}$. The pressure was kept constant at 1 bar using the Berendsen isotropic pressure coupling algorithm with a time constant of 1 ps. ${ }^{58}$ The LINCS algorithm was used to constrain bond lengths, ${ }^{59}$ while SETTLE was applied to constrain the internal degrees of freedom of the water molecules, ${ }^{60}$ enabling a time step of $2 \mathrm{fs}$ in the classical MD simulations. A $1.0 \mathrm{~nm}$ cut-off was used for non-bonded Van der Waals' interactions, which were modeled with Lennard-Jones potentials. Coulomb interactions were computed with the smooth particle mesh Ewald method, ${ }^{61}$ using a $1.0 \mathrm{~nm}$ real space cut-off and a grid spacing of $0.12 \mathrm{~nm}$. The relative tolerance at the real space cut-off was set to $10^{-5}$. All simulations were performed with the Gromacs-4.5 molecular dynamics program. ${ }^{51}$

From the classical MD ensemble statistically independent snapshots were extracted to serve as starting points for the cavity QM/MM simulations. Inspection of the molecular orbitals involved in the $S_{0}$ to $S_{1}$ transition suggests that the optical excitation is localized on the fused three-ring system and hardly involves orbitals on the perpendicular benzene ring. Therefore, we constructed a QM/MM model with only the fused ring system inside the QM region. Restricted Hartree-Fock with a 3-21G basis set ${ }^{62}$ was used to model the ground state $\left(\mathrm{S}_{0}\right)$ electronic structure, energies and gradients, while Configuration Interaction, truncated at single electron excitations, was used to model the excited state $\left(S_{1}\right)$. The rest of the molecule and the solvent were modelled with the Amber03 force field ${ }^{52}$, as in the classical MD simulations. The bond connecting the QM and MM subsystems was replaced by a constraint ${ }^{59}$ and the QM part was capped with a hydrogen atom. The force on the cap atom was distributed over the two atoms of the bond. ${ }^{20}$ The QM system experienced the Coulomb field of all MM atoms within a $1.6 \mathrm{~nm}$ cut-off sphere around the Rhodamine atoms and Lennard- 


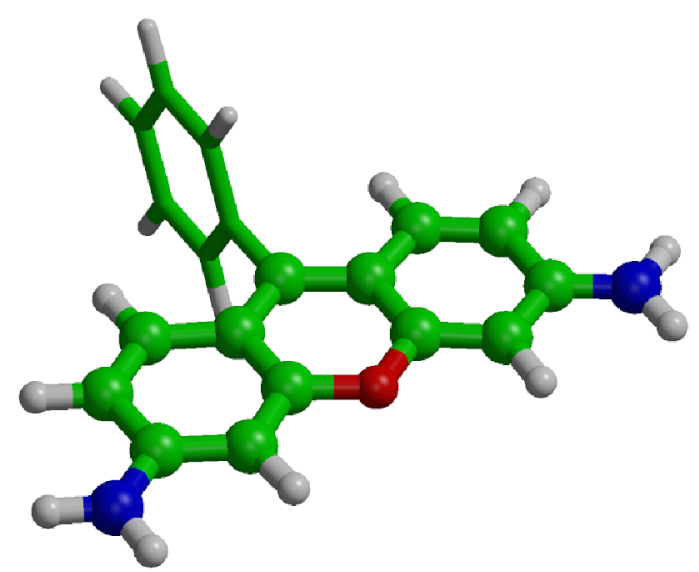

Figure 4: Rhodamine QM/MM model system. The QM atoms, treated at the RHF/3-21G and CIS/3-21G levels of theory for the ground $\left(S_{0}\right)$ and excited states $\left(S_{1}\right)$, respectively, are shown in ball-and-stick represenation, while the MM atoms, modeled with the Amber03 force field ${ }^{52}$, are shown as sticks. The hydrogen link atom introduced along the bond on the QM/MM interface is not shown, nor are the 3684 TIP3P water molecules. ${ }^{57}$

Jones interactions between $\mathrm{MM}$ and QM atoms were added. The time step was reduced to 0.1 fs and the temperature was kept at $300 \mathrm{~K}$ with the v-rescale thermostat $\left(\tau_{\mathrm{T}}=0.1 \mathrm{ps}\right) .{ }^{63}$ Prior to the molecular dynamics simulations of Rhodamine in the cavity, the snapshots were further equilibrated for 5 ps at the RHF/321G//Amber03 QM/MM level.

The cavity frequency $\left(\omega_{\text {cav }}\right)$ was tuned at the excitation maximum of a single Rhodamine molecule in water, evaluated at the QM/MM level (4.115 eV, see below). The cavity volume was chosen to yield a Rabi splitting of $\sim 0.5 \mathrm{eV}$ when there are 800 molecules in the cavity. A Rabi splitting of several hundreds of $\mathrm{meV}$ is in line with recent experiments. ${ }^{64-67}$ The number of molecules coupled to the cavity was varied from 1 to 800 . Cavity lifetimes exceeding $100 \mathrm{fs}$ are possibe, ${ }^{68}$ and we ran our simulations for up to $10 \mathrm{fs}$ in such cavity. Because in the assumed cavity there would virtually be no decay within the length of the simulation, we neglected the spontaneous deactivation of the cavity photon in our simulations. In addition to simulations of ensembles with up to 800 molecules inside a cavity, we also performed simulations of a single Rhodamine molecule in the excited state at the CIS/3-21G level of theory. All QM/MM simulations were performed with the QM/MM interface between Gromacs-4.5 ${ }^{51}$ and Gaussian09. ${ }^{56}$

The Hellmann-Feynman forces acting on the nuclei require the derivative of the transition dipole mo- 
ment with respect to atomic displacements (equation 16). Although in some programs, such as TeraChem, ${ }^{69}$ such derivatives are available, they would need to be implemented in Gaussian09. ${ }^{56}$ However, because no large conformational changes were observed in neither $S_{0}$ nor $S_{1}$ simulations, we follow Sisto et al. and approximate the transition dipole moment by a Taylor expansion around the $\mathrm{S}_{0}$ equilibrium conformation $\left(\mathbf{R}_{0}\right)$, truncated at first order: ${ }^{45}$

$$
\begin{aligned}
\boldsymbol{\mu}^{g \rightarrow e}(\mathbf{R})= & \boldsymbol{\mu}^{g \rightarrow e}\left(\mathbf{R}_{0}\right)+\sum_{i}^{n} \frac{\partial}{\partial x_{i}} \boldsymbol{\mu}^{g \rightarrow e}\left(\mathbf{R}_{0}\right)\left(x_{i}-x_{0, i}\right)+ \\
& \sum_{i}^{n} \frac{\partial}{\partial y_{i}} \boldsymbol{\mu}^{g \rightarrow e}\left(\mathbf{R}_{0}\right)\left(y_{i}-y_{0, i}\right)+\sum_{i}^{n} \frac{\partial}{\partial z_{i}} \boldsymbol{\mu}^{g \rightarrow e}\left(\mathbf{R}_{0}\right)\left(z_{i}-z_{0, i}\right)
\end{aligned}
$$

The derivatives were obtained through least-squares fitting with 5,000 QM/MM snapshots. The quality of the fit was tested by comparing the predicted and calculated transition dipole moment in 1,000 snapshots that were not used for the fitting (Supporting Information)

Absorption and emission spectra were calculated from QM/MM trajectories in the ground $\left(\mathrm{S}_{0}\right)$ and excited states $\left(\mathrm{S}_{1}\right)$, respectively. These spectra were composed from the $\mathrm{S}_{1}-\mathrm{S}_{0}$ energy gaps $\left(\Delta E_{i}\right)$ by superposition of Gaussian functions: ${ }^{70}$

$$
I(E)=\sum_{i=1}^{N} f_{i} \exp \left[-\left(E-\Delta E_{i}\right)^{2} / 2 \sigma^{2}\right]
$$

where $I(E)$ is the intensity as a function of excitation energy $E, f_{i}$ the oscillator strength of the transition with excitation energy $\Delta E_{i}$, and the sum runs over $1000 \mathrm{QM} / \mathrm{MM}$ snapshots. A width of $\sigma=0.05 \mathrm{eV}$ was chosen for the convolution.

\section{Results and Discussion}

We now briefly illustrate our cavity QM/MM MD method by performing simulations of Rhodamine molecules strongly coupled to a cavity tuned at the QM/MM absorption maximum of these molecules. We have chosen the simplest Rhodamine system, rather than the more complex fluorophores that have been used in cavity experiments so far, such as tetra-(2,6-t-butyl)phenol-porphyrin zinc (4TBPPZn), ${ }^{71}$ 1,10-diethyl-3,30-bis(4sulfobutyl)-5,50,6,60-tetrachlorobenzimidazolocarbocyanine (TDBC) J-aggregates, 5-(4-(dibutylamino)-benzylidene)- 

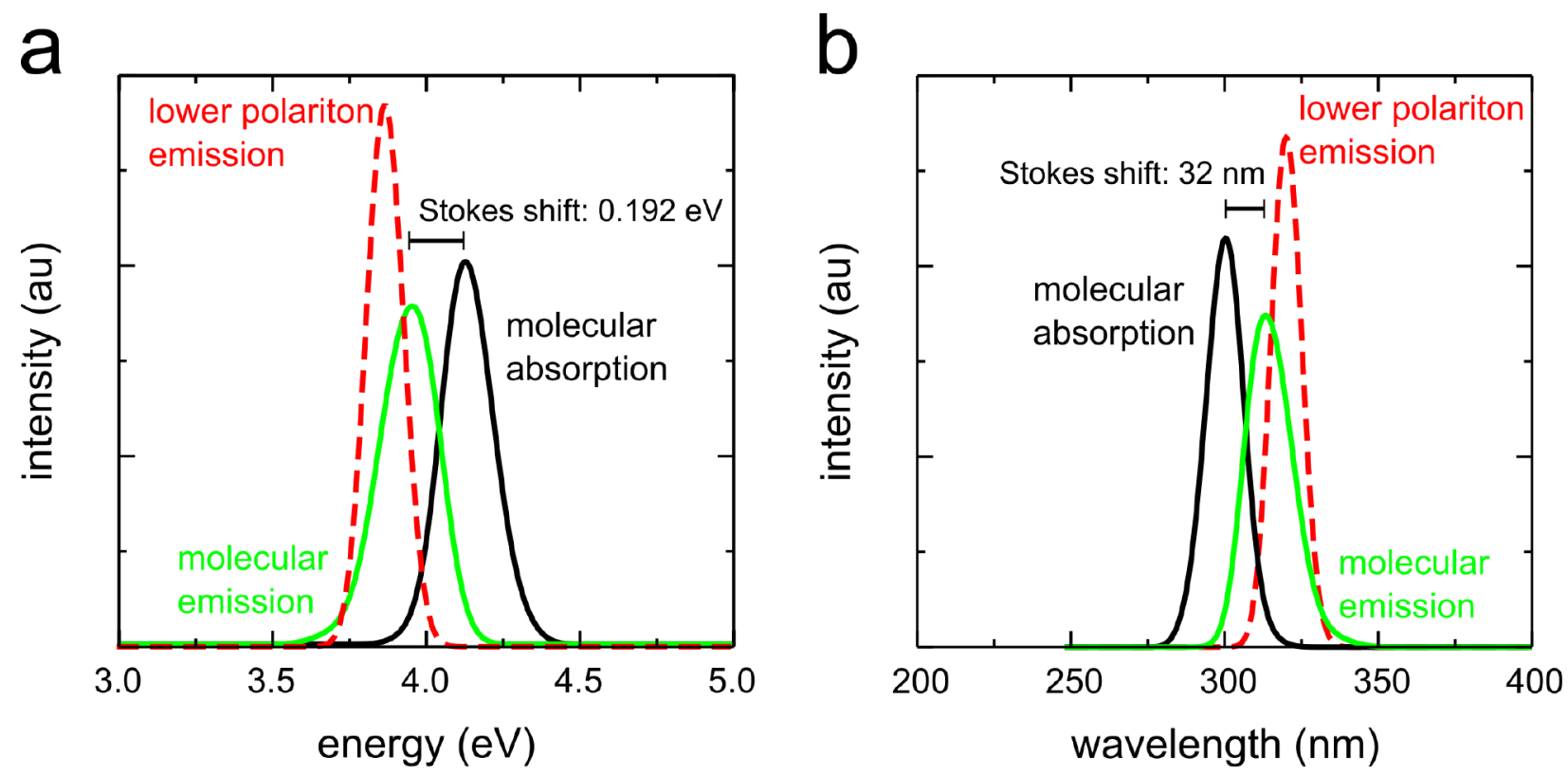

Figure 5: Absorption (black) and emission (green) spectra obtained from constant temperature QM/MM MD simulations of a single Rhodamine molecule in water on an eV (a) and nm scale (b). Emission from the lower polaritonic state with 800 molecules strongly coupled to the cavity photon is shown as a red dashed line in panel (a).

1,3-dimethylpyrimidine-2,4,6(1H,3H,5H)-trione (BDAB) or fluorescin. ${ }^{64}$ Although our choice for a simpler molecule precludes a direct comparison to previous cavity experiments, the general effect of the light-matter interaction can be investigated in atomic detail. Moreover, we have only simulated the dynamics after excitation into the lower polariton. Although with our model also the relaxation from the upper polariton could be simulated in atomic detail, we leave this aspect of polariton dynamics ${ }^{72}$ for future work, in which we combine simulation and transient spectroscopy to address this issue.

Figure 5 shows the calculated absorption and emission spectra of the 'bare' (i.e., outside of the cavity) Rhodamine in water. The Stokes shift of $0.192 \mathrm{eV}(32 \mathrm{~nm})$ agrees with the difference in the energy gap between the optimized geometries in the ground and excited states $(0.198 \mathrm{eV})$. Compared to popular Rhodamine variants, such as Rhodamine-6G or Sulfur-Rhodamine101, the Stokes shift is similar, but the spectra are too blue shifted. The blue shift is likely a consequence of (i) the much smaller conjugated system in the QM/MM model, (ii) the small basis set and (iii) the truncated Configuration Interaction scheme. Furthermore, whereas in cavities the chromophores are usualy embedded in polymer matrices (e.g. poly-vinyl- 
a

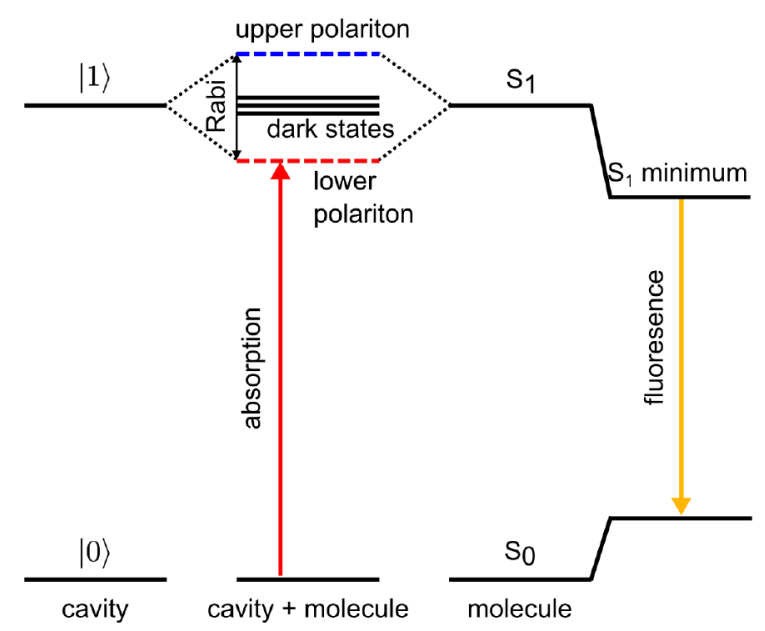

b

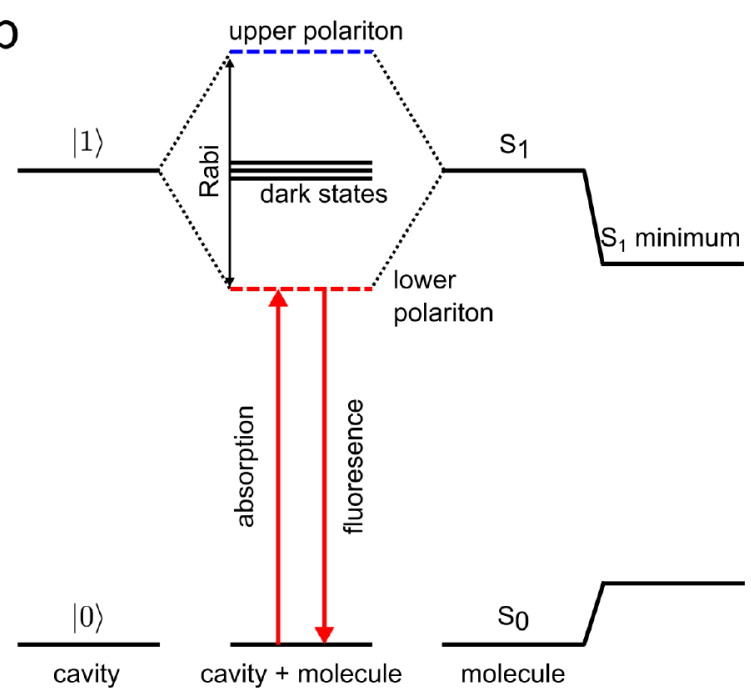

Figure 6: Effect of the Stokes shift on polariton stability. If the coupling, manifested by Rabi splitting, is small (a), the minimum on the $S_{1}$ potential energy surface (Stokes shifted minimum) is below the polariton energy. In this situation, the lower polariton can decay via localization of the excitation on a single molecule and subsequent relaxation into the $\mathbf{S}_{1}$ minimum of that molecule. In contrast, if the coupling, and hence Rabi splitting, is large enough, the polariton is the lowest energy state and the system can remain there until spontaneous emission takes place (b).

alcohol, PVA), we used water as the solvent instead. The choice for water as a molecular environment and thermal bath, rather than a polymer, was motivated by the slow convergence of polymer melts in MD simulations. Nevertheless, although the specific interactions with the polar groups may be different compared to PVA, the absorption maximum of Rhodamine is not very sensitive to solvent. Furthermore, including an explicit water environment goes significantly beyond the neglect or implicit treatment of an environment in phenomenological models of strong coupling, such as the Lindblad formalism. ${ }^{73}$

The stability of the polariton depends on both the Q-factor of the cavity and on the excited state lifetime of the molecules. Whereas for both cavities and atoms, the lifetime, or equivalently decay rate, can be directly inferred from the spectral widths, molecular absorption is dominated by vibronic progression and hence the line width does not provide an accurate measure for lifetime. Furthermore, in contrast to atoms, molecules relax after photo-excitation by adjusting their geometry and solvent environment to the excited state electron density. Because this relaxation is accompanied by a reduction of the energy gap, it increases the emission wavelength, known as the Stokes shift. To investigate the relation between the Stokes shift and 

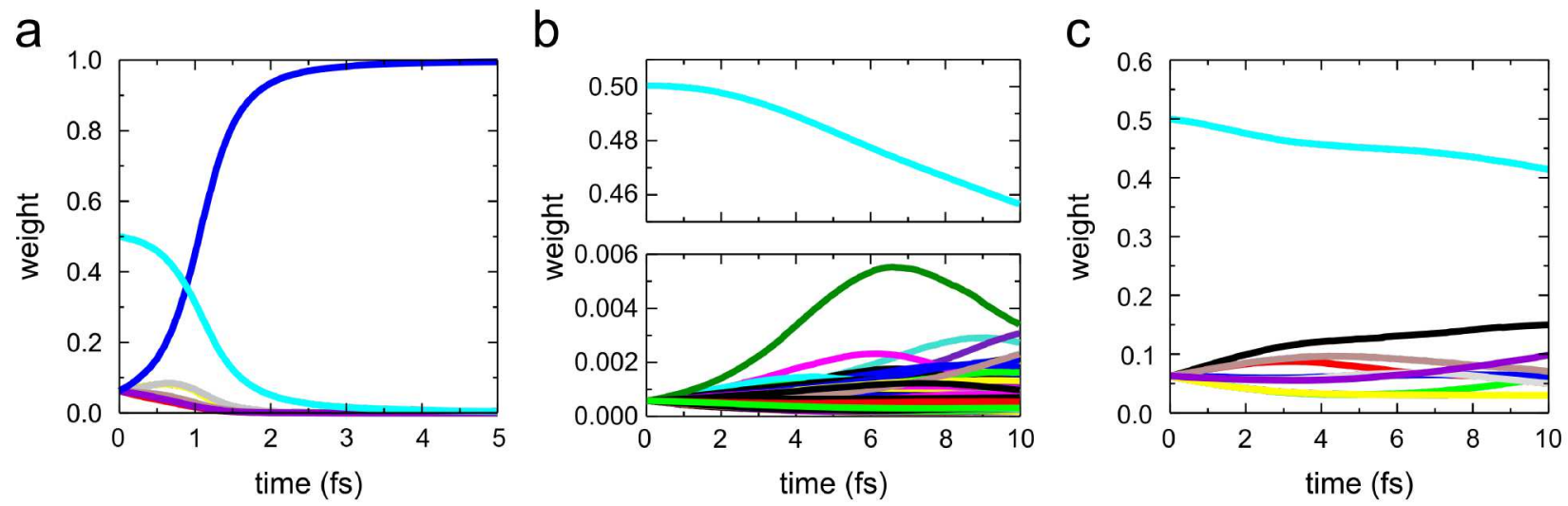

Figure 7: Time evolution of the lower polariton during $10 \mathrm{fs}$ of MD simulation of (a) 8, (b) 800 Rhodamine molecules strongly coupled to confined light in a microcavity and (c) 8 molecules coupled to a nanocavity with a 100 times smaller mode volume. Plotted are the weights of the excitations on each molecule $\left(\left|\beta_{i}^{\mathrm{LP}}\right|^{2}\right.$ in all colours $)$ and of the cavity photon $\left(\left|\alpha^{\mathrm{LP}}\right|^{2}\right.$ in cyan).

the light-matter coupling, we performed MD simulations at various coupling strengths (Figure 6). Because the coupling is proportional to the square root of the number of molecules (Equation 9), we varied the latter to control the energy level of the lower (and upper) polariton in these simulations.

First, we performed simulations of ensembles of up to 800 molecules, all starting from the same snapshot, but with different initial atomic velocities sampled randomly from a Maxwell-Boltzmann distribution at $300 \mathrm{~K}$. The time-evolution of the polaritons with 8 and 800 Rhodamines is plotted in Figure 7 . With only 8 molecules in the cavity the Rabi-split is $\sim 0.05 \mathrm{eV}$ and the lower polariton has a higher energy $(\sim 4.09 \mathrm{eV})$ than the $S_{1}$ minimum of the Rhodamine (3.98 eV, Figures 5 and 6). As a consequence, the excitation localizes within femtoseconds onto a single Rhodamine molecule (Figure 7a), which then relaxes into the minimum on the $S_{1}$ potential energy surface. In contrast, increasing the coupling by ten-fold, achieved with hundred times more molecules, brings the lower polaration below the $S_{1}$ minimum of Rhodamine. Indeed, as shown in Figure 5a the emission maximum of the lower polariton is below the emission maximum of 'bare' Rhodamine. In this situation, the excitation remains delocalized over all molecules and cavity photon (Figure 7b).

Since our simulations are valid for molecules coupled to both optical cavities and surface plasmons, the results of the simulations provide a tentative explanation for our previous observation that the polarization of light emitted from polaritons composed of photoactive molecules strongly interacting with propagating 
surface plasmons (PSP), has a dependency on the Stokes shift of the molecules. ${ }^{74}$ Because PSPs on flat metal films are only supported with $p$-polarization, PSP emission is always $p$-polarized when detected perpendicular to the metal surface. ${ }^{75}$

In our previous work ${ }^{74}$ we have shown theoretically that without molecular dynamics, the emission from a molecule-PSP polaritonic state should also be purely $p$-polarized. While this is indeed observed if the molecules have a large Stokes shift, unpolarized polariton emission was observed for molecules with negligible Stokes shift (e.g., TDBC J-aggregates). Based on the time-evolution of the weights in our simulations we interpret these differences as follows: Molecules with small Stokes shift remain part of the polariton, but the thermal fluctuations of their environments break the initial symmetry of the polariton (i.e. $\beta(t) \neq \beta(0)$ and $\alpha(t) \neq \alpha(0)$, Figure 7b-c). Due to these fluctuations, in combination with the variations in the transition dipole moments of the molecules, the polarization of the polariton emission becomes randomized at later times. In contrast, if the Stokes shift is large, the polariton has a very short lifetime, because the excitation rapidly localizes onto a single molecule, from where unpolarized molecular emission (i.e., normal fluorescence) occurs (Figure 7a). The latter is also consistent with the experimental observation of an additional emission peak that nicely matches the molecular fluorescence spectrum. ${ }^{74}$ In this case the polariton life time is too short for the weights to alter significantly and we therefore conjecture that the emission from the polariton is largely $p$-polarized.

Combined, the results from the simulations and the previous experiments ${ }^{74}$ suggest a generalization of Kasha's rule, ${ }^{76}$ which states that emission occurs predominantly from the lowest excited state, to polaritons. Polariton emission dominates if the polariton energy is below the $S_{1}$ minimum of the molecules. Otherwise, the excitation rapidly localizes onto a single molecule from where emission occurs, unless there are competing non-adiabatic deactivation channels. Thus, despite the collective excitation of many molecules, a polariton behaves like a super-molecule. Our observations furthermore suggest that by engineering the Stokes shift of the chromophores, the flow of excitation energy can be controlled inside cavities, which might prove useful for artificial light harvesting.

In addition to increasing the number of molecules, the light-matter interaction can also be enhanced by decreasing the mode volume (Equation 4). Experimentally, smaller mode volumes can be achieved if, instead of Fabry-Pérot cavities, surface plasmons are used to confine light. ${ }^{23}$ Surface plasmons are collective bosonic excitations of the electron gas on the surface of metals. Because of their confinement to the metal 
surface, very small mode volumes can be realized. ${ }^{66,74,77,78}$ With volumes far below the diffraction limit of the light, the strong coupling regime can already be achieved with few molecules and recently Rabi splitting was even observed with only a single molecule in a plasmonic nano-cavity. ${ }^{78}$ The main disadvantage of plasmonic nano-cavities, however, is a much lower Q-factor due to increased losses via radiative and nonradiative decay channels.

Nevertheless, we also investigated the effect of reducing the mode volume by a factor of 100 (Equations 4 and 9) for 8 molecules in the cavity (technically now a plasmonic nano-cavity), keeping an infinite Q-factor by artificially suppressing deactivion of the cavity photon. As with 800 molecules, the excitation does not localize in the smaller cavity, but remains delocalized over all molecules and cavity photon (Figure 7c). A plot showing $100 \mathrm{fs}$ of dynamics is included in Supporting Information. Compared to the simulations with 800 molecules in the cavity the strength of the interaction with confined light as experienced by the single molecules is 10 times larger (Equation 4). Although at a timescale of $10 \mathrm{fs}$ this did not lead to an observable difference in the conformational sampling of the molecules under strong coupling, shrinking mode volume to increase the coupling strength is not equivalent to adding more molecules. Therefore, whether mimicking the effect of a large number of molecules through a higher effective coupling strength, as is often done in coupled oscillator models, ${ }^{23}$ is valid, needs to be further investigated. ${ }^{79,80}$

Next, we selected ensembles of up to 800 molecules (plus environment) from the single molecule QM/MM trajectories and coupled these ensembles to the cavity. In contrast to the previous simulations, the molecules not only have different starting velocities, but also different inital coordinates. However, this does not change the behaviour. As shown in Figure 8a, the polariton rapidly deactivates if the excited state mimimum of Rhodamine is below the polariton energy. In contrast, when the polariton has a lower energy than the $S_{1}$ minimum, which was achieved by increasing the number of molecules (Figure $8 \mathrm{~b}$ ) again or by decreasing the mode volume of the cavity (Figure 8c), the excitation remains delocalized and the polariton lifetime is only limited by the cavity Q-factor.

Summarizing, the results of our MD simulations suggest that if the light-matter coupling is strong enough to bring the energy of the lower polariton below the $S_{1}$ minimum of the molecules, the excitation remains delocalized until the cavity photon decays. During the polariton lifetime, the polariton evolves because the contributions of molecules $\left(\beta_{i}^{\mathrm{LP}}\right)$ and photon $\left(\alpha^{\mathrm{LP}}\right)$ in the superposition (Equation 8$)$ change with time due to variations in the molecular environment that affect the dipole moments and excitation ener- 
a

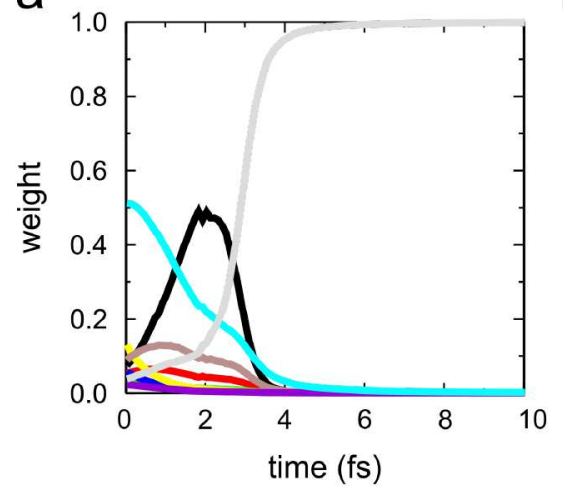

b

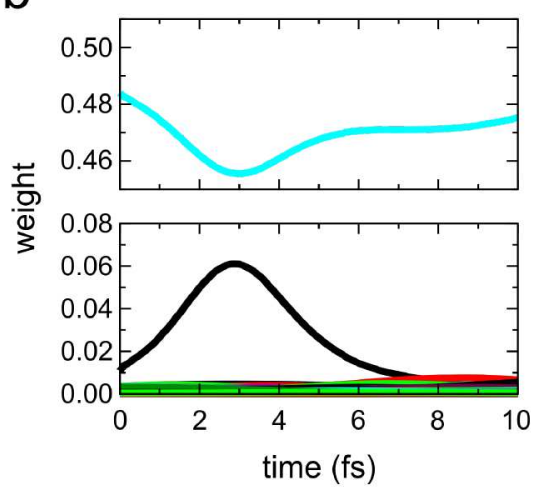

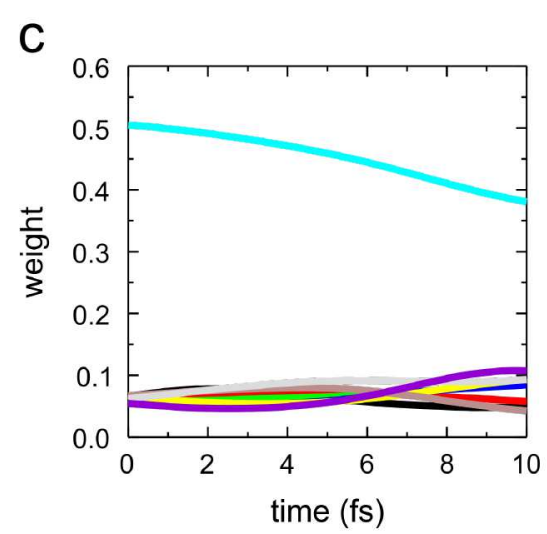

Figure 8: Time evolution of the lower polariton during $10 \mathrm{fs}$ MD simulation of (a) 8, (b) 800 Rhodamine molecules strongly coupled to confined light in a microcavity and (c) 8 molecules coupled to a nanocavity with a 100 times smaller mode volume. Plotted are the weights of of the excitations of each molecule $\left(\left|\beta_{i}^{\mathrm{LP}}\right|^{2}\right.$ all colours) and of the cavity photon $\left(\left|\alpha^{\mathrm{LP}}\right|^{2}\right.$ cyan). The difference with respect to Figure 7 is that both initial atomic positions and velocities are different for the molecules and their environments.

gies of the molecules. These variations not only break the initial symmetry of the polariton, but should also lead to experimentally observable changes in the polarization of photons emitted at the polaritonic energy. ${ }^{74}$

\section{Conclusions and Outlook}

We have extended the treatment of molecular dynamics on hybrid light-matter potential energy surfaces ${ }^{17,18}$ to realistic molecules interacting with their environment using QM/MM excited state molecular dynamics. ${ }^{38}$ We have shown that we can simulate the dynamics of large ensembles of molecules strongly coupled with a single cavity photon. Because the simulations provide atomistic insights, we expect our method to aid the interpretation of recent experiments on molecules in cavities ${ }^{13}$ and yield new experimentally verifiable hypotheses. While in the simulations presented here, the absolute accuracy of the underlying level of quantum chemistry theory was low and the conditions deviated from typical cavity experiments, these aspects of our model can be easily improved (at the cost of additional computational effort) when comparing to experiments in the future. Synergy between experiment and simulations will be essential not only to validate our model but also to make a beginning in understanding what effects strong coupling can have on molecular 
dynamics and reactivity. Ultimately, our model has the potential to pave the way for systematic design and optimization of cavities or plasmonic nanostructures to selectively alter well-known photochemical reactions.

\section{Acknowledgements}

This work was supported by the Academy of Finland (Grants 290677 to JJT and GG, and 258806 to GG) as well as the European Research Council (ERC-2011-AdG-290981) and the European Union Seventh Framework Programme (FP7-PEOPLE-2013-CIG-618229, JF). We thank T. T. Heikkilä, D. Morozov, C. F. Burmeister and Oleg Blyednov for valuable discussions. We acknowledge the Center for Scientific Computing (CSC-IT Center for Science) for computational resources and thank N. Runeberg and S. Ilvonen in particular for their help with running the simulations on the Cray XC40. Finally, we acknowledge PRACE for awarding us access to resource Curie based in France at GENCI.

\section{Supporting Information}

The Supporting Information contains the force field parameters for the Rhodamine model, plots comparing the predicted transition dipole moment to the ab initio dipole moment and 100 fs dynamics of the lower polariton with 8 Rhodamine molecules plus solvent inside the cavity. This information is available free of charge via the Internet at http://pubs.acs.org.

\section{References}

(1) Nørskov, J. K.; Bligaard, T.; Rossmeisl, J.; Christensen, C. H. Towards the computational design of solid catalysts. Nature Chemistry 2009, 1, 37-146.

(2) Röthlisberger, D.; Khersonsky, O.; Wollacott, A. M.; Jiang, L.; DeChancie, J.; Betker, J.; Gallaher, J. L.; Althoff, E. A.; Zanghellini, A.; Dym, O.; Albeck, S.; Houk, K. N.; Tawfik, D. S.; Baker, D. Kemp elimination catalysts by computational enzyme design. Nature 2008, 453, 190-195. 
(3) Fu, Q.; McKenzie, T. G.; Ren, J. M.; Tan, S.; Nam, E.; Qiao, G. G. A novel solid state photocatalyst for living radical polymerization under UV irradiation. Sci. Rep. 2016, 6, 20779.

(4) Polli, D.; Altoe, P.; Weingart, O.; Spillane, K. M.; Manzoni, C.; Brida, D.; Tomasello, G.; Orlandi, G.; Kukura, P.; Mathies, R. A.; Garavelli, M.; Cerullo, G. Conical intersection dynamics of the primary photoisomerization event in vision. Nature 2010, 467, 440-443.

(5) Croce, R.; Amerongen, H. V. Natural strategies for photosynthetic light harvesting. Nat. Chem. Biol. 2014, 10, 492-501.

(6) Wöhri, A. B.; Katona, G.; Johansson, L. C.; Fritz, E.; Malmerberg, E.; Andersson, M.; Vincent, J.; Eklund, M.; Ewald, F.; Cammarata, M.; Wulff, M.; Davidsson, J.; Groenhof, G.; Neutze, R. Lightinduced structural changes in a photosynthetic reaction center caught by Laue diffraction. Science 2010, 328, 630-633.

(7) Assion, A.; Baumert, T.; M, B.; Brixner, T.; Kiefer, B.; Seyfried, V.; Strehle, M.; Gerber, G. Control of Chemical Reactions by Feedback-Optimized Phase-Shaped Femtosecond Laser Pulses. Science 1998, 282, 919-922.

(8) Prokhorenko, V. I.; Nagy, A. M.; Waschuk, S. A.; Brown, L. S.; Birge, R. R.; Miller, R. J. D. Coherent Control of Retinal Isomerization in Bacteriorhodopsin. Science 2006, 313, 1257-1261.

(9) Sussman, B. J.; Townsend, D.; Ivanov, M. Y.; Stolow, A. Dynamic Stark Control of Photochemical Processes. Science 2006, 314, 278-281.

(10) Hutchison, J. A.; Schwartz, T.; Genet, C.; Devaux, E.; Ebbesen, T. W. Modifying Chemical Landscapes by Coupling to Vacuum Fields. Angew. Chem. Int. Ed. 2012, 51, 1592-1596.

(11) Coles, D. M.; Somaschi, N.; Michetti, P.; Clark, C.; Lagoudakis, P. G.; Savvidis, P. G.; Lidzey, D. G. Polariton-mediated energy transfer between organic dyes in a strongly coupled optical microcavity. Nature Materials 2014, 13, 712-719.

(12) Zhong, X.; Chervy, T.; Wang, S.; George, J.; Thomas, A.; Hutchison, J. A.; Devaux, E.; Genet, C.; Ebbesen, T. W. Non-Radiative Energy Transfer Mediated by Hybrid Light-Matter States. Angew. Chem. Int. Ed. 2016, 55, 6202-6206. 
(13) Ebbesen, T. W. Hybrid Light-Matter States in a Molecular and Material Science Perspective. Acc. Chem. Res. 2016, 49, 2403-2412.

(14) Mazza, L.; Kéna-Cohen, S.; Michetti, P.; Rocca, G. C. L. Microscopic theory of polariton lasing via vibronically assisted scattering. Phys. Rev. B. 2013, 88, 075321.

(15) Cẃik, J. A.; Reja, S.; Littlewood, P. B.; Keeling, J. Polariton condensation with saturable molecules dressed by vibrational modes. Eur. Phys. Lett. 2014, 105, 47009.

(16) Spano, F. C. Optical microcavities enhance the exciton coherence length and eliminate vibronic coupling in J-aggregates. J. Chem. Phys. 2015, 142, 184707.

(17) Galego, J.; Garcia-Vidal, F. J.; Feist, J. Cavity-Induced Modifications of Molecular Structure in the Strong-Coupling Regime. Phys. Rev. X 2015, 5, 041022.

(18) Galego, J.; Garcia-Vidal, F. J.; Feist, J. Suppressing photochemical reactions with quantized light fields. Nat. Comm. 2016, 7, 13841.

(19) Warshel, A.; Levitt, M. Theoretical studies of enzymatic reactions: Dielectric, electrostatic and steric stabilization of carbonium ion in the reaction of lysozyme. J. Mol. Biol. 1976, 103, 227-249.

(20) Groenhof, G. Introduction to QM/MM simulations. Meth. Mol. Biol. 2013, 924, 12489-12491.

(21) Mabuchi, H.; Doherty, A. C. Cavity Quantum Electrodynamics: Coherence in Context. Science 2002, $298,1372-1377$.

(22) Purcell, E. M. Spontaneous emission probabilities at radio frequencies. Phys. Rev. 1946, 69, 681.

(23) Törmä, P.; Barnes, W. L. Strong coupling between surface plasmon polaritons and emitters: a review. Rep. Prog. Phys. 2015, 78, 013901.

(24) De Liberato, S. Light-Matter Decoupling in the Deep Strong Coupling Regime: The Breakdown of the Purcell Effect. Phys. Rev. Lett. 2014, 112, 016401.

(25) Flick, J.; Ruggenthaler, M.; Appel, H.; Rubio, A. Cavity Born-Oppenheimer Approximation for correlated Electron-Nuclear-Photon Systems. J. Chem. Theory Comput. in press, DOI: 10.1021/acs.jctc.6b01126 2017, 
(26) Flick, J.; Ruggenthaler, M.; Appel, H.; Rubio, A. Atoms and Molecules in Cavities: From Weak to Strong Coupling in QED Chemistry. Proc. Natl. Acad. Sci. USA in press DOI: 10.1073/pnas.1615509114 2017,

(27) Jaynes, E. T.; Cummings, F. W. Comparison of quantum and semiclassical radiation theories with to the beam maser. Proc. IEEE 1963, 51, 89-109.

(28) Tavis, M.; Cummings, F. W. Approximate solutions for an N-molecule radiation-field Hamiltonian. Phys. Rev. 1969, 188, 692-695.

(29) Houdré, R.; Stanley, R. P.; Ilegems, M. Vacuum-field Rabi splitting in the presence of inhomogeneous broadening: Resolution of a homogeneous linewidth in an inhomogeneously broadened system. Phys. Rev. A 1996, 53, 2711-2715.

(30) Bennett, K.; Kowalewski, M.; Mukamel, S. Novel photochemistry of molecular polaritons in optical cavities. Faraday Discuss. 2016, 194, 259-282.

(31) Kowalewski, M.; Bennett, K.; Mukamel, S. Cavity Femtochemistry: Manipulating Nonadiabatic Dynamics at Avoided Crossings. J. Phys. Chem. Lett. 2016, 7, 2050-2054.

(32) Kowalewski, M.; Bennett, K.; Mukamel, S. Non-adiabatic dynamics of molecules in optical cavities. J. Chem. Phys. 2016, 144, 054309.

(33) Herrera, F.; Spano, F. C. Cavity-controlled chemistry in molecular ensembles. Phys. Rev. Lett. 2016, $116,238301$.

(34) Csehi, A.; Halás, G. J.; Cederbaum, L.; Vibók, Á. Competition between Light-Induced and Intrinsic Nonadiabatic Phenomena in Diatomics. J. Chem. Phys. Lett. 2017, 8, 1624-1630.

(35) Warshel, A. Bicycle-Pedal Model for First Step in Vision Process. Nature 1976, 260, 679-683.

(36) McCammon, J. A.; Gelin, B. R.; Karplus, M.; Wolynes, P. G. Hinge-bending mode in lysozyme. Nature 1976, 262, 325-326.

(37) Lindorff-Larsen, K.; Piana, S.; Dror, R.; Shaw, D. E. How fast-folding proteins fold. Science 2011, $334,517-520$. 
(38) Boggio-Pasqua, M.; Burmeister, C. F.; Robb, M. A.; Groenhof, G. Photochemical reactions in biological systems: probing the effect of the environment by means of hybrid quantum chemistry/molecular mechanics simulations. Phys. Chem. Chem. Phys. 2012, 14, 7912-7928.

(39) Fabiano, E.; Groenhof, G.; Thiel, W. Approximate switching algorithms for trajectory surface hopping. Chem. Phys. 2008, 351, 111-116.

(40) Groenhof, G.; Bouxin-Cademartory, M.; Hess, B.; de Visser, S. P.; Berendsen, H. J. C.; Olivucci, M.; A. E. Mark, M. A. R. Photoactivation of the photoactive yellow protein: Why photon absorption triggers a trans-to-cis isomerization of the chromophore in the protein. J. Am. Chem. Soc 2004, 124, $4228-4232$.

(41) Pande, K.; Hutchison, C. D. M.; Groenhof, G.; Aquila, A.; Robinson, J. S.; Tenboer, J.; Basu, S.; Boutet, S.; DePonte, D. P.; Liang, M.; White, T. A.; Zatsepin, N. A.; Yefanov, O.; Morozov, D.; Oberthuer, D.; Gati, C.; Subramanian, G.; James, D.; Zhao, Y.; Koralek, J.; Brayshaw, J.; Kupitz, C.; Conrad, C.; Roy-Chowdhury, S.; Coe, J. D.; Metz, M.; Xavier, P. L.; Grant, T. D.; Koglin, J. E.; Ketawala, G.; Fromme, R.; Srajer, V.; Henning, R.; Spence, J. C. H.; Ourmazd, A.; Schwander, P.; Weierstall, U.; Frank, M.; Fromme, P.; Barty, A.; Chapman, H. N.; Moffat, K.; van Thor, J. J.; Schmidt, M. Femtosecond Structural Dynamics Drives the Trans-to-Cis Isomerization in Photoactive Yellow Protein. Science 2016, 352, 725-729.

(42) Roos, B. O. . Theoretical studies of electronically excited states of molecular systems using multiconfigurational perturbation theory. Acc. Chem. Res. 1999, 32, 137-144.

(43) Runge, E.; Gross, E. K. U. Density-Functional Theory for Time-Dependent Systems. Phys. Rev. Lett 1984, $52,997-1000$.

(44) Molina, P. A.; H.Li,; Jensen, H. Intraprotein Electrostatics Derived from First Principles: Divide-andConquer Approaches for QM/MM Calculations. J. Comput. Chem. 2003, 24, 1971-1979.

(45) Sisto, A.; Glowacki, D. R.; Martinez, T. J. Ab Initio Nonadiabatic Dynamics of Multichromophore Complexes: A Scalable Graphical-Processing-Unit-Accelerated Exciton Framework. Acc. Chem. Res. 2014, 47, 2857-2866. 
(46) Landau, L. Zur theorie der energieubertragung. II. Phys. Z. Sowjetunion 1932, 2, 1-13.

(47) Zener, C. Non-adiabatic crossing of energy levels. Proc. R. Soc. A 1932, 137, 696-702.

(48) Desouter-Lecomte, M.; Lorquet, J. Nonadiabatic interactions in unimolecular decay. IV. Transition probability as a function of the Massey parameter. J. Chem. Phys. 1979, 71, 4391-4403.

(49) Hammes-Schiffer, S.; Tully, J. C. Proton transfer in solution: molecular dynamics with quantum transitions. J. Chem. Phys. 1994, 101, 4657-4667.

(50) Vukovic, L.; Burmeister, C. F.; Kral, P.; Groenhof, G. Control Mechanisms of Photoisomerization in Protonated Schiff Bases. J. Phys. Chem. Lett. 2013, 4, 1005-1101.

(51) Hess, B.; Kutzner, C.; van der Spoel, D.; Lindahl, E. GROMACS 4: Algorithms for Highly Efficient, Load-Balanced, and Scalable Molecular Simulation. J. Chem. Theory Comput. 2008, 4, 435-447.

(52) Duan, Y.; Wu, C.; Chowdhury, S.; Lee, M. C.; Xiong, G. M.; Zhang, W.; Yang, R.; Cieplak, P.; Luo, R.; Lee, T.; Caldwell, J.; Wang, J. M.; Kollman, P. A point-charge force field for molecular mechanics simulations of proteins based on condensed-phase quantum mechanical calculations. $J$. Comput. Chem. 2003, 24, 1999-2012.

(53) Bayly, C. I.; Cieplak, P.; Cornell, W. D.; Kollman, P. A. A well-behaved electrostatic potential bsed method using charge restraints for deriving atomic charges - the RESP model. J. Phys. Chem. 1993, 97, 10269-10280.

(54) Becke, A. D. A new mixing of Hartree-Fock and local density-functional theories. J. Chem. Phys. 1993, 98, 1372.

(55) Lee, C. T.; Yang, W. T.; Parr, R. G. Development of the Colle-Salvet correlation-energy formula into a functional of the electron density. Phys. Rev. B 1988, 37, 785-789.

(56) Frisch, M. J.; Trucks, G. W.; Schlegel, H. B.; Scuseria, G. E.; Robb, M. A.; Cheeseman, J. R.; Scalmani, G.; Barone, V.; Mennucci, B.; Petersson, G. A.; Nakatsuji, H.; Caricato, M.; Li, X.; Hratchian, H. P.; Izmaylov, A. F.; Bloino, J.; Zheng, G.; Sonnenberg, J. L.; Hada, M.; Ehara, M.; Toyota, K.; Fukuda, R.; Hasegawa, J.; Ishida, M.; Nakajima, T.; Honda, Y.; Kitao, O.; Nakai, H.; 
Vreven, T.; Montgomery, J. A., Jr.; Peralta, J. E.; Ogliaro, F.; Bearpark, M.; J.Heyd, J.; Brothers, E.; Kudin, K. N.; Staroverov, V. N.; Kobayashi, R.; Normand, J.; Raghavachari, K.; Rendell, A.; Burant, J. C.; Iyengar, S. S.; Tomasi, J.; Cossi, M.; Rega, N.; Millam, J. M.; Klene, M.; Knox, J. E.; Cross, J. B.; Bakken, V.; Adamo, C.; Jaramillo, J.; Gomperts, R.; Stratmann, R. E.; Yazyev, O.; Austin, A. J.; Cammi, R.; Pomelli, C.; Ochterski, J. W.; Martin, R. L.; Morokuma, K.; Zakrzewski, V. G.; Voth, G. A.; Salvador, P.; Dannenberg, J. J.; Dapprich, S.; Daniels, A. D.; Farkas, Ö.; Foresman, J. B.; Ortiz, J. V.; Cioslowski, J.; Fox, D. J. Gaussian 09 Revision D.1. Gaussian Inc. Wallingford CT 2009.

(57) Jorgensen, W. L.; Chandrasekhar, J.; Madura, J. D.; Impey, R. W.; Klein, M. L. Comparison of simple potential functions for simulatin liquid water. J. Chem. Phys. 1983, 79, 926-935.

(58) Berendsen, H.; Postma, J.; van Gunsteren, W.; la, A. D.; Haak, J. Molecular dynamics with coupling to an external bath. J. Chem. Phys. 1984, 81, 3684-3690.

(59) Hess, B.; Bekker, H.; Berendsen, H. J. C.; Fraaije, J. G. E. M. LINCS: A linear constraint solver for molecular simulations. J. Comput. Chem. 1997, 18, 1463-1472.

(60) Miyamoto, S.; Kollman, P. A. SETTLE: An analytical version of the SHAKE and RATTLE algorithms for rigid water molecules. J. Comp. Chem. 1992, 18, 1463-1472.

(61) Essmann, U.; Perera, L.; Berkowitz, M. L.; Darden, T.; Lee, H.; Pedersen, L. G. A smooth particle mesh Ewald potential. J. Chem. Phys 1995, 103, 8577-8592.

(62) Ditchfield, R.; Hehre, W. J.; Pople, J. A. Self-Consistent Molecular-Orbital Methods. IX. An Extended Gaussian-Type Basis for Molecular-Orbital Studies of Organic Molecules. J. Chem. Phys. 1971, 54, $724-728$.

(63) Bussi, G.; Donadio, D.; Parrinello, M. Canonical sampling through velocity rescaling. J. Chem. Phys. 2007, 126, 014101.

(64) George, J.; Wang, S.; Chervy, T.; Canaguier-Durand, A.; Schaeffer, G.; Lehn, J.-M.; Hutchison, J. A.; Genet, C.; Ebbesen, T. W. Ultra-strong coupling of molecular materials: spectroscopy and dynamics. Faraday Discuss. 2015, 178, 281-294. 
(65) Schwartz, T.; Hutchison, J. A.; Leonard, J.; Genet, C.; Haacke, S.; Ebbesen, T. W. Polariton Dynamics under Strong Light-Molecule Coupling. ChemPhysChem 2013, 14, 125-131.

(66) Zengin, G.; Wersäll, M.; Nilsson, S.; aand M. Käll, T. J. A.; Shegai, T. Realizing Strong Light-Matter Interactions between Single-Nanoparticle Plasmons and Molecular Excitons at Ambient Conditions. Phys. Rev. Lett. 2015, 114, 157401.

(67) Melnikau, D.; Esteban, R.; Savateeva, D.; Sánchez-Iglesias, A.; Grzelczak, M.; Schmidt, M. K.; LizMarzán, L. M.; Aizpurua, J.; Rakovich, Y. P. Rabi Splitting in Photoluminescence Spectra of Hybrid Systems of Gold Nanorods and J-Aggregates. J. Phys. Chem. Lett. 2016, 7, 354-362.

(68) Dietrich, C. P.; Steude, A.; Tropf, L.; Schubert, M.; Kronenberg, N. M.; Ostermann, K.; Höfling, S.; Gather, M. C. An exciton-polariton laser based on biologically produced fluorescent protein. Sci. Adv. 2016, 2, e1600666.

(69) Titov, A. V.; Ufimtsev, I. S.; Luehr, N.; Martińez, T. J. Generating Efficient Quantum Chemistry Codes for Novel Architectures. J. Chem. Theory Comput. 2013, 9, 213-221.

(70) Schäfer, L. V.; Groenhof, G.; Klingen, A. R.; Ullmann, G.; Boggio-Pasqua, M.; Robb, M. A.; Grubmüller, H. Photoswitching of the Fluorescent Protein asFP595: Mechanism, Proton Pathway, and Absorption Spectra. Angew. Chem. Int. Ed. 2007, 46, 9347-9438.

(71) Lidzey, D. G.; Bradley, D. D. C.; Skolnick, M. S.; Virgili, T.; Walker, S.; Whittaker, D. M. Strong exciton-photon coupling in an organic semiconductor microcavity. Nature 1998, 53-55.

(72) Litinskaya, M.; Reineker, P.; Agranovich, V. M. Fast polariton relaxation in strongly coupledorganic microcavities. J. Lumin. 2004, 110, 364-372.

(73) Lindblad, G. On the generators of quantum dynamical semigroups. Comm. Math. Phys. 1976, 48, 119-130.

(74) Baieva, S.; Hakamaa, O.; Groenhof, G.; Heikkilä, T. T.; Toppari, J. J. Dynamics of strongly coupled modes between surface plasmon polaritons and photoactive molecules: the effect of the Stokes shift. ACS Photonics 2017, 4, 28-37. 
(75) Kretschmann, E. The angular dependence and the polarization of light emitted by surface plasmons on metals due to roughness. Opt. Commun. 1972, 5, 331-336.

(76) Kasha, M. Characterization of Electronic Transitions in Complex Molecules. Disc. Faraday Soc. 1950, 9, 14-19.

(77) Santhosh, K.; Bitton, O.; Chuntonov, L.; Haran, G. Vacuum Rabi splitting in a plasmonic cavity at the single quantum emitter limit. Nat. Comm. 2016, 7, 11823.

(78) Chikkaraddy, R.; de Nijs, B.; Benz, F.; Barrow, S. J.; Scherman, O. A.; Rosta, E.; Demetriadou, A.; Fox, P.; Hess, O.; Baumberg, J. J. Single-molecule strong coupling at room temperature in plasmonic nanocavities. Nature 2016, 535, 127-130.

(79) Sáez-Blázquez, R.; Feist, J.; Fernández-Domínguez, A. I.; García-Vidal, F. J. Enhancing Photon Correlations through Plasmonic Strong Coupling. Arxiv 2017, 1701.08964v1, 1-5.

(80) Cortese, E.; Lagoudakis, P.; De Liberato, S. Cavity quantum electrothermodynamics with rotational degrees of freedom. Arxiv 2017, 1701.01689v1, 1-4. 


\section{Table of Content Graphic}

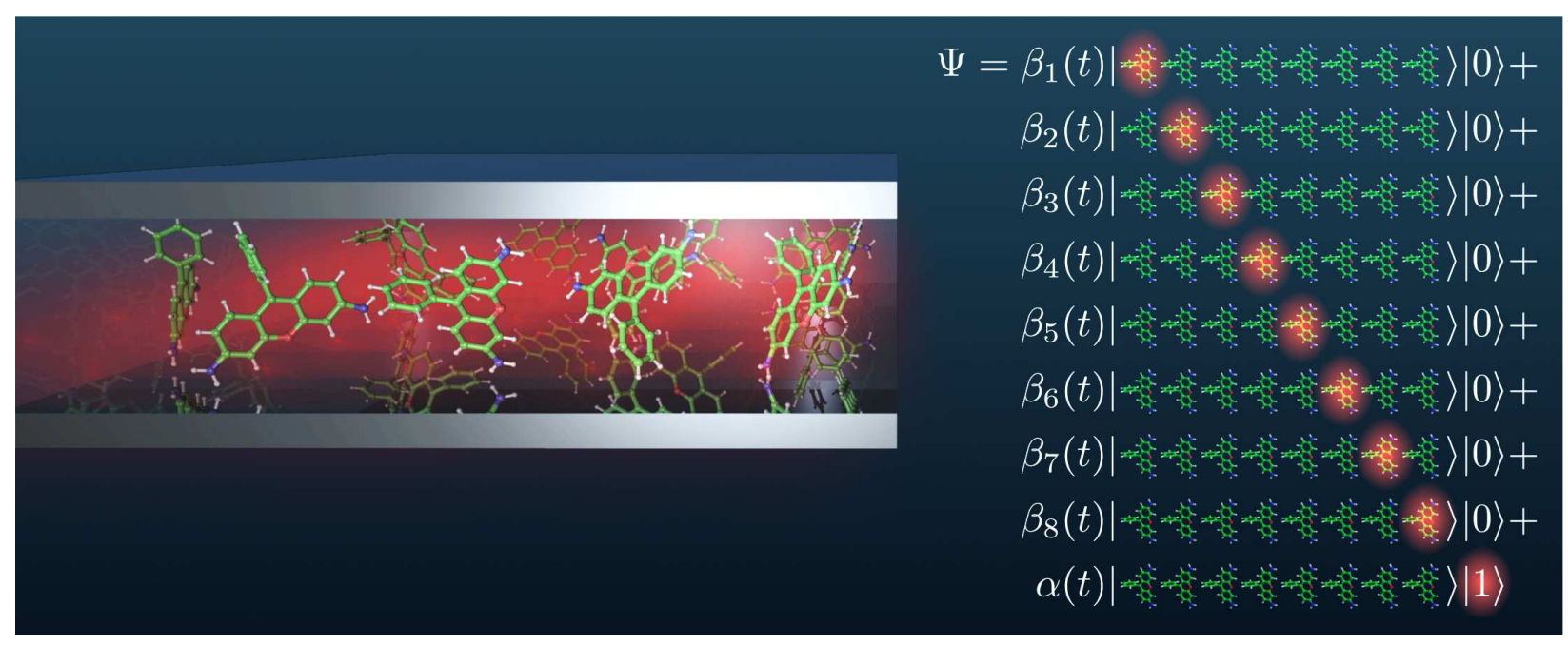

